\title{
The Impact of Intra-Uterine Manipulators on Outcome and Recurrence Patterns of Endometrial Cancer Patients Undergoing Minimally Invasive Surgery
}

Ido Laskov

Tel Aviv Sourasky Medical Center https://orcid.org/0000-0002-5061-0670

Nadav Michaan

Tel Aviv University https://orcid.org/0000-0001-7987-404X

Liron Kogan

Tel Aviv Sourasky Medical Center

\section{Xing Zeng}

McGill University and McGill University Health Centre

\section{Shannon Salvador}

Tel Aviv Sourasky Medical Center https://orcid.org/0000-0002-8061-375X

\section{Kris Jardon}

McGill University and McGill University Health Centre

\section{Susie Lau}

Tel Aviv Sourasky Medical Center

\section{Lucy Gilbert}

McGill University and McGill University Health Centre https://orcid.org/0000-0001-8605-5472

Walter H. Gotlieb ( $\sim$ walter.gotlieb@mcgill.ca )

Tel Aviv Sourasky Medical Center https://orcid.org/0000-0001-6227-6369

\section{Roy Kessous}

Tel Aviv Sourasky Medical Center

\section{Research Article}

Keywords: endometrial cancer, uterine manipulator, hysterectomy, recurrence

Posted Date: June 24th, 2021

DOl: https://doi.org/10.21203/rs.3.rs-538902/v1

License: (a) This work is licensed under a Creative Commons Attribution 4.0 International License. Read Full License 
Page $2 / 21$ 


\section{Abstract}

\section{Purpose}

To evaluate the use of an intrauterine manipulator on the oncologic outcome of women who had minimally invasive surgery for endometrial cancer.

\section{Methods}

Retrospective analysis of consecutive patients who were operated with or without the use of an intrauterine manipulator. Univariate and multivariate analysis were used to adjust for possible confounders.

Results

699 patients were included, of whom 220 (32.8\%) were operated with an intrauterine manipulator. The median follow-up was 44 months (range, 29-67). Disease-free survival was similar between groups. 19 $(8.8 \%)$ patients had positive cytology in the manipulator group vs. $21(4.4 \%)$ in the comparison group $(p=0.02)$. Total recurrence rate was similar between the groups $(12.3 \%$ vs. $11.9 \% ; p=0.8)$. Vaginal vault recurrence was the most common site of recurrence with higher incidence in the manipulator group (4.5\% vs. $1.3 \% ; p=0.007)$. Sub-group analysis of patients who did not receive adjuvant treatment showed higher recurrence rate $(8.3 \%$ vs. $3 \% ; p=0.023)$ and worse disease-free survival $(p=0.01)$ for the manipulator group. After controlling for other variables, the use of a manipulator did not affect the risk of recurrence for the whole cohort $(\mathrm{HR}, 1.28 ; 95 \% \mathrm{Cl}, 0.7-2.1, \mathrm{p}=0.3)$ and for the sub-group of patients who did not receive adjuvant treatment $(\mathrm{HR}, 2.47 ; 95 \% \mathrm{Cl}, 0.8-7, \mathrm{p}=0.08)$.

Conclusion

The use of a manipulator during surgery for endometrial cancer increases the risk of positive cytology as well as vaginal vault recurrences, but it does not reduce the disease-free and overall survival of patients.

\section{Introduction}

Currently, the gold standard treatment for patients diagnosed with endometrial cancer is based primarily on staging surgery followed by adjuvant treatment if indicated [1]. After the primary surgery, patients are divided into risk groups (low, intermediate, and high) that will guide physicians as to the recommended adjuvant treatment if needed. The division into the different risk groups is based on multiple factors including patients' age, histologic subtype, tumor grade, staging of disease and presence of lymphovascular space invasion [1-3]. Approximately $6-13 \%$ of all patients with endometrial cancer will develop recurrent disease, the majority during the first 3 years after a primary diagnosis with primary location in the vaginal vault [4]. 
Two minimally invasive (MIS) approaches are used for endometrial cancer staging surgery, total laparoscopic hysterectomy (TLH) and robotically assisted total laparoscopic hysterectomy (RH) [5]. Compared to laparotomy, the benefits of minimal invasive approach hysterectomy for endometrial cancer, include reduced blood loss, shorter recuperation time, lower cost, less use of pain medication, faster recovery, similar or fewer post-operative adverse events, and better quality of life up to 6 months after surgery [6]. In addition, no significant difference exists in cancer recurrence or outcome between MIS and laparotomy cases, indicating comparable oncologic safety of MIS [7].

For proper uterine manipulation during minimal invasive hysterectomy, various uterine manipulators are used with or without an intrauterine segment (usually a screw, a rod or a balloon) [8]. The intra-uterine component is inserted into the uterine cavity in order to allow surgeons manipulation of the uterus for better exposure. In addition to the intrauterine component, some of the uterine manipulators have a colpotomizer (cervical cap) that assists in identifying the anatomic landmarks such as the vaginal fornices and allow to increase the distance between the ureter and the vaginal vault [9].

Nevertheless, some surgeons are avoiding the use of the intrauterine component in cases of endometrial cancer, for concern of iatrogenic spillage, uterine perforation, and the potential risk of retrograde dissemination of cancer cells into the peritoneal cavity or the lymphatic spaces. In order to avoid this potential spread, some surgeons choose either not to use a uterine manipulator at all or to use an uterine manipulator with a colpotomizer but without an intrauterine segment [10].

The aim of this study was to evaluate the effect of the use of an intrauterine manipulator on the recurrence rate and outcome of patients undergoing minimal invasive staging surgery for endometrial cancer.

\section{Methods}

\section{Study population and setting}

A retrospective cohort study, identifying all consecutive patients who underwent a minimal invasive hysterectomy for endometrial cancer at the McGill university tertiary-care hospitals during the years 2008-2016. All surgeries were performed by laparoscopic or robotic approach by 6 gynecologic oncology surgeons. MIS for staging of endometrial cancer included peritoneal cytology, total hysterectomy and salpingo-oophorectomy, with or without pelvic and/or para-aortic lymph node dissection (LND) or sentinel lymph node detection (SLND). In cases where the pre-operative histology was papillary serous or clear cell, surgical staging also included omentectomy, examination of the entire abdomino-pelvic cavity and retroperitoneal spaces. During the study period, routine coagulation of the fallopian tubes at the beginning of the procedure to avoid possible intraperitoneal spread of disease was not performed. Patients who had uterine perforation, morcellation, or conversion to laparotomy were excluded from the study.

\section{Uterine manipulators}


During the study, uterine manipulators that were used were either a Clermont Ferrand manipulator (Karl Storz, Tuttlingen, Germany), RUMI uterine manipulator with a Koh colpotomizer system (Cooper Surgical, Trumbull, CT, USA) or a ZUMI uterine manipulator (Cooper Surgical, Trumbull, CT, USA). In other cases, either a uterine manipulator was not used or an adjustment was made to the Hohl uterine manipulator (Storz, El Segundo, CA) so that it included only the handle with its colpotomizer without its intra-uterine component as described previously by our group [10]. The decision as to the type of intrauterine manipulator or lack of it was left to the discretion of the surgeon.

\section{Clinical information}

Data was collected from the department's digital records, including operative reports, pathology reports, chemotherapy records, radiation oncology reports, laboratory results, and radiology reports were reviewed to determine baseline and perioperative characteristics until the end of follow-up period or death for each patient.

Baseline characteristics included age, body mass index and medical comorbidities. Perioperative data included the surgical procedure with the type of uterine manipulator or colpotomizer used, stage of malignancy based on the 2009 FIGO for endometrial carcinoma [11], grade, histology, depth of myometrial invasion, lymph-vascular space invasion, fallopian tube and ovarian involvement, lower uterine segment and cervix involvement, lymph node involvement with total count, uterine size, tumor size and classification of pelvic cytology. Pelvic cytology was routinely obtained as follows: the suction irrigator was used to infuse saline into the pelvis following placement of the uterine manipulator. The manipulator was used to displace the uterus anteriorly while the saline was retrieved using the suction irrigator device; volume was not standardized. Because the risk of tumor dissemination may be related to tumor size and volume, measurements provided in the gross and microscopic sections of pathology reports were used to determine the maximum diameter of tumor and to calculate tumor volume if threedimensional measurements were provided. Post-operative complications were collected in real time.

For those patients who had recurrence, disease free survival (DFS) was defined as time from last treatment to the diagnosis of recurrence. Overall survival (OS) was defined as time from diagnosis to time of last follow-up or death. Recurrences were diagnosed clinically or radiologically. All vaginal recurrences were biopsy proven. During the surveillance period, routine follow-up examinations were performed at intervals of 4 months during the first two years, followed by every 6 months for up to 5 years, and then yearly until the end of the follow-up period or death. To assess the effect of different uterine manipulator types on LVSI, positive cytology and patient's outcome, patients were divided into two groups. The first group (Intrauterine manipulator group) included patients who were operated using a uterine manipulator that necessitates a cervical os dilation prior to the placement of the intrauterine rod (Clermont Ferrand, RUMI or ZUMI) and a second group (No intrauterine manipulator group) of patients that were operated either without a uterine manipulator or using the intravaginal part of a Hohl uterine manipulator without the intrauterine manipulator, as previously published (10).

\section{Statistical analysis}


Statistical analysis was done using SPSS software (IBM, SPSS statistics, Version 25, 2017. IBM® corp. Armonk, NY, USA). All statistical analyses were 2 sided and $\mathrm{P}<0.05$ was considered significant. Continuous variables were compared using either one-way ANOVA or Kruskal- Wallis and Mann-Whitney tests according to distribution of variables. Chi squared test or Fishers' exact test were used to compare categorical variables. Disease free survival and overall survival were calculated using Kaplan Meier estimator and compared with log rank test with reverse censoring method. Univariate as well as multivariate cox regression analysis was used to evaluate the association between each of the predictors and disease-free survival and overall survival.

\section{Results}

During the study period we identified 782 patients who underwent minimal invasive hysterectomy for endometrial intraepithelial neoplasia (EIN) and newly diagnosed endometrial cancer. Eighty three patients $(10.6 \%)$ were excluded from the study: 54 patients with EIN, 22 patients required conversion to laparotomy (2.8\%), most of those required a simple mini-laparotomy at the end of the procedure in order to remove the uterus that could not be removed intact via the vagina (utero-vaginal disproportion) and 9 patients had a large uterus that was morcellated within an endobag placed via the vagina (1.1\%). After exclusion, six-hundred and sixty-nine patients were included in the analysis and the clinicopathologic characteristics are presented in Table 1. The mean patient age was 64 years (SD 11), and the mean body mass index (BMI) was $31.7 \mathrm{~kg} / \mathrm{m}^{2}$ (SD 8.6). Six hundred and thirteen patients (88\%) underwent robotic assisted hysterectomy and 86 patients (12\%) underwent laparoscopic hysterectomy. In four hundred and eighty-four patients (69\%) the procedure included pelvic lymph node dissection (PLND) and for 203 patients (29\%), para aortic lymph node dissection was performed in addition to PLND. The most common uterine manipulator used was the Hohl retractor $(60 \%)$ without intrauterine component, followed by the Clermont Ferrand manipulator (19\%). 
Table 1

Patient characteristics and surgical outcomes $(n=699)$.

\section{Characteristics}

Age, mean (SD), y

BMI, mean (SD), $\mathrm{kg} / \mathrm{m} 2$

Surgery mode, $n(\%)$

Manipulator type, n (\%)
Robotic

Laparoscopic

Surgical procedure, $\mathrm{n}(\%)$

Histology, n (\%)

Staget, $\mathrm{n}(\%)$

Tumor gradet, $\mathbf{n}(\%)$

$\mathrm{TH}+\mathrm{BSO}$
$\mathrm{TH}+\mathrm{BSO}+\mathrm{PLND}$
$\mathrm{TH}+\mathrm{BSO}+\mathrm{PLND}+\mathrm{PaLND}$

Hohl

$\mathrm{KF}$

ZUMI

$\mathrm{RUMI} /$ Koh

None

Endometrioid

Serous

Clear

Other

la

Ib

II

III

IV

1
Total

( $n=699)$

64.5 (11)

$31.7(8.6)$

613 (88)

86 (12)

12 (1.7)

484 (69.3)

203 (29)

$420(60)$

136 (19)

62 (9)

22 (3)

59 (9)

546 (78)

91 (13)

19 (3)

$43(6)$

416 (59)

$113(16)$

$42(6)$

$116(17)$

$12(2)$

301 (43)

SD, standard deviation; BMI, body mass index; TH, total hysterectomy; BSO, bilateral salpingooophorectomy; PLND, pelvic lymph node dissection; PaLND, para aortic lymph node dissection; CF, Clermont Ferrand; RTx, radiotherapy; CTx, chemotherapy.

†Tumor stage is presented according to the 2009 International Federation of Gynecology and Obstetrics stage and histologically classified and graded according to the World Health Organization. 
Myometrial invasion, $\mathrm{n}(\%)$

No

Less than half

More than half

Lymph vascular invasion, $\mathrm{n}$ (\%)

Lymph node retrieval number, mean (SD)

Positive Lymph node metastasis, $\mathbf{n}(\%)$

Positive cytology, n (\%)

Adjuvant therapy, n (\%)
None

RTx only

CTx only

Combined RTx + CTx
$62(9)$

$582(83)$

$55(8)$

$206(29)$

$12.3(8.8)$

$84(12)$

$40(5.7)$

$369(53)$

$124(17)$

$26(4)$

$180(23)$

$45(29-67)$

Follow up time, median (range), month

SD, standard deviation; BMI, body mass index; TH, total hysterectomy; BSO, bilateral salpingooophorectomy; PLND, pelvic lymph node dissection; PaLND, para aortic lymph node dissection; CF, Clermont Ferrand; RTx, radiotherapy; CTx, chemotherapy.

tTumor stage is presented according to the 2009 International Federation of Gynecology and Obstetrics stage and histologically classified and graded according to the World Health Organization.

The majority of patients had stage I disease $(n=528,75 \%)$ and endometrioid histology $(n=546,78 \%)$, whereas 205 patients (29\%) had grade 3 disease. Three hundred and thirty patients (44\%) with moderate to high risk factors received adjuvant treatment, either radiotherapy, chemotherapy or combination of the two. The median follow-up time was 44 (range 29-67) months.

Out of the 699 eligible patients, 220 (31.4\%) underwent surgery with the support of an intrauterine manipulator (Intrauterine manipulator group) and in 479 patients $(68.6 \%)$ no intrauterine manipulator was used (No intrauterine manipulator group). The distribution is presented in Fig. 1. There were no significant differences between the two groups with regard to age at surgery, BMI, histologic type, FIGO stage, LVSI, tumor size and lymph node retrieval number (Table 2). Women in the intrauterine manipulator group had significantly more grade 1 disease $(50 \%$ vs. $39 \%, p=0.01)$ and positive cytology $(8.8 \%$ vs. $4.4 \%, p=0.02$ ) compared to women in the no intrauterine manipulator group. Eighty-seven patients $(39.5 \%)$ in the intrauterine manipulator group compared to $243(50.7 \%)$ in the no intrauterine manipulator 
were classified to be intermediate/high risk for recurrence and received adjuvant treatment $(p=0.006) .27$ patients (12.3\%) recurred in the intrauterine manipulator group, compared to $57(11.9 \%)$ patients in the no intrauterine manipulator group $(\mathrm{p}=0.88)$. Vaginal vault recurrence was the most common site of recurrence, occurring in $10(4.5 \%)$ patients in the intrauterine manipulator group vs. $6(1.3 \%)$ patients in the no intrauterine manipulator group $(p=0.007)$. Nine patients $(4.1 \%)$ in the intrauterine manipulator group had pelvic recurrences vs. 9 pelvic recurrences $(1.9 \%)$ in the no intrauterine manipulator group $(p=$ 0.08). Lower limb lymphedema was the most prevalent post-operative complication, occurring in 16 (7.3\%) patients of the manipulator group compared to $12(2.5 \%)$ patients in the no manipulator group ( $p$ $=0.003$ ). Less common complications were wound and urinary infections but did not show statistical difference between the groups. Median DFS and OS were not reached and were not statistically different between the two groups. 
Table 2

Patient characteristics and surgical outcomes with patterns of recurrence $(n=699)$ and subgroup analysis for patients who did not receive adjuvant treatment $(n=369)$.

\section{Characteristics}

\section{Total Cohort}

\section{Intra-uterine}

manipulator
No intra-uterine
manipulator
P value

\begin{tabular}{|c|c|c|c|c|}
\hline & & $\mathrm{n}=\mathbf{2 2 0}$ & $n=479$ & \\
\hline Age, mean (SD), yea & & $64.9(11)$ & $64.3(11)$ & 0.605 \\
\hline BMI, mean (SD), kg/ & & $31.2(8)$ & $32.0(9)$ & 0.392 \\
\hline Histology, n (\%) & Endometrioid & $179(81.4)$ & 367 (76.8) & 0.156 \\
\hline & Serous & $26(11.8)$ & $65(13.6)$ & \\
\hline & Clear & $5(2.3)$ & $14(2.9)$ & \\
\hline & Other & $10(4.5)$ & $32(6.7)$ & \\
\hline Staget, n (\%) & la & $144(65.5)$ & $271(56.7)$ & 0.071 \\
\hline & $\mathrm{lb}$ & $24(10.9)$ & 89 (18.6) & \\
\hline & II & $13(5.9)$ & $29(6.1)$ & \\
\hline & III & $39(17.7)$ & $77(16.1)$ & \\
\hline Tumor gradet, n & 1 & $111(50.5)$ & 189 (39.5) & 0.01 \\
\hline & 2 & $54(24.5)$ & $139(29.1)$ & \\
\hline & 3 & $55(25.0)$ & 150 (31.4) & \\
\hline Lymph vascular inva & ion, n (\%) & 69 (31.4) & 137 (28.7) & 0.478 \\
\hline $\begin{array}{l}\text { Lymph node retrieva } \\
\text { (SD) }\end{array}$ & number, mean & $13.8(10.8)$ & $11.6(7.6)$ & 0.101 \\
\hline Positive Lymph nod & netastasis, n (\%) & $24(11.0)$ & $60(12.7)$ & 0.525 \\
\hline Positive cytology, n & & $19(8.8)$ & $21(4.4)$ & 0.022 \\
\hline Tumor size, median & ange), cm & $3.2(1.9)$ & $3.3(1.9)$ & 0.199 \\
\hline Uterine size, median & ange), cm & $8.2(1.9)$ & $7.8(2.2)$ & 0.005 \\
\hline Adjuvant therapy, n & Total & $87(39.5)$ & $243(50.7)$ & 0.006 \\
\hline & RTx only & $29(13)$ & $95(20)$ & 0.033 \\
\hline
\end{tabular}

SD, standard deviation; BMI, body mass index.

†Tumor stage is presented according to the 2009 International Federation of Gynecology and

Obstetrics stage and histologically classified and graded according to the World Health Organization. 


\begin{tabular}{|c|c|c|c|c|}
\hline \multicolumn{2}{|l|}{ Characteristics } & \multirow{2}{*}{$\begin{array}{l}\text { Intra-uterine } \\
\text { manipulator }\end{array}$} & \multirow{2}{*}{$\begin{array}{l}\begin{array}{l}\text { No intra-uterine } \\
\text { manipulator }\end{array} \\
15(3)\end{array}$} & \multirow{2}{*}{$\begin{array}{l}P \\
\text { value } \\
0.226\end{array}$} \\
\hline & CTx only & & & \\
\hline & $\begin{array}{l}\text { Combined RTx + } \\
\text { CTx }\end{array}$ & $47(21)$ & $133(28)$ & 0.072 \\
\hline Recurrence, $n(\%)$ & & $27(12.3)$ & $57(11.9)$ & 0.888 \\
\hline \multirow[t]{6}{*}{ Site, n (\%) } & Vaginal vault & $10(4.5)$ & $6(1.3)$ & 0.007 \\
\hline & Pelvic & $9(4.1)$ & $9(1.9)$ & 0.087 \\
\hline & Abdominal & $8(3.6)$ & $22(4.6)$ & 0.563 \\
\hline & $\begin{array}{l}\text { Retroperitoneal } \\
\text { LN }\end{array}$ & $8(3.6)$ & $20(4.2)$ & 0.736 \\
\hline & Lung & $4(1.8)$ & $19(4.0)$ & 0.139 \\
\hline & Multiple site & $11(5.0)$ & $22(4.6)$ & 0.814 \\
\hline \multirow{4}{*}{$\begin{array}{l}\text { Complications, } n \\
(\%)\end{array}$} & Lymphedema & $16(7.3)$ & $12(2.5)$ & 0.003 \\
\hline & Wound infection & $9(4.1)$ & $9(1.9)$ & 0.087 \\
\hline & Urinary infection & $5(2.3)$ & $10(2.1)$ & 0.875 \\
\hline & Vaginal cuff & $1(0.5)$ & $6(1.3)$ & 0.325 \\
\hline Death, $n(\%)$ & & $30(13.6)$ & $54(11.3)$ & 0.378 \\
\hline \multicolumn{5}{|l|}{ Subgroup Analysis } \\
\hline & & $n=133$ & $n=236$ & \\
\hline \multicolumn{2}{|c|}{ Lymph vascular invasion, $\mathrm{n}(\%)$} & $10(7.5)$ & $13(5.6)$ & 0.504 \\
\hline \multicolumn{2}{|c|}{ Positive cytology, n (\%) } & $6(4.5)$ & $3(1.3)$ & 0.077 \\
\hline \multicolumn{2}{|l|}{ Recurrence (\%) } & $11(8.3)$ & $7(3)$ & 0.023 \\
\hline \multicolumn{2}{|c|}{ Vaginal vault recurrence, $\mathrm{n}(\%)$} & $7(5.3)$ & $2(0.8)$ & 0.012 \\
\hline \multicolumn{5}{|c|}{ SD, standard deviation; BMI, body mass index. } \\
\hline
\end{tabular}

To further assess the effect of uterine manipulation on patient's outcome we have performed a subgroup analysis of all patients who did not receive adjuvant treatment (Table 2). In the intrauterine manipulator group, $11(8.3 \%)$ patients recurred as compared to $7(3 \%)$ in the no intrauterine manipulator group $(\mathrm{p}=$ $0.02)$, with a $5.3 \%$ rate of vaginal vault recurrence compared to $0.8 \%(p=0.01)$. Median DFS was not reached however, in a Kaplan-Meyer survival analysis, patients in whom no intrauterine manipulator was 
used had significantly better DFS (Fig. 2B, $p=0.01$ ). When all the patients were included, whether they received adjuvant treatment or not, no significant differences in DFS could be observed between the groups (Fig. 2A).

To better assess the association between the use of intrauterine manipulator and recurrence rate as well as outcome of patients, a multivariate analysis was performed (Table 3). Figo stage, grade, LVSI, positive cytology and adjuvant treatment were all independently associated with risk of disease recurrence and decreased OS. The use of a uterine manipulator was not associated with the risk of relapse of endometrial cancer (Hazard ratio, 1.28; $95 \%$ confidence interval, $0.7-2.1, p=0.3$ ). The use of a uterine manipulator did not reach statistical significance for risk of relapse on a second multivariate analysis performed on the subgroup of patients who did not receive adjuvant treatment (Hazard ratio, 2.47; 95\% confidence interval, 0.87-7.01, $p=0.08$ ) (Table 3). 
Table 3

Factors associated with patient outcome in multivariate analysis.

\begin{tabular}{|c|c|c|c|c|}
\hline & \multicolumn{2}{|l|}{ Disease Free Survival } & \multicolumn{2}{|l|}{ Overall Survival } \\
\hline & Hazard ratio $(95 \% \mathrm{Cl})$ & $P$ value & Hazard ratio $(95 \% \mathrm{Cl})$ & $P$ value \\
\hline \multicolumn{5}{|l|}{ Total Cohort } \\
\hline Age & $1.05(0.98-1.02)$ & 0.62 & $1.06(1.03-1.08)$ & $<0.001$ \\
\hline Stage $1 \mathrm{~A}$ & 1 & 0.053 & 1 & 0.06 \\
\hline 1B & $2.16(0.9-4.7)$ & 0.003 & $2.26(0.94-5.42)$ & 0.009 \\
\hline 2 & $3.8(1.6-9.3)$ & $<0.001$ & $3.51(1.36-9.04)$ & $<0.001$ \\
\hline 3 & $4.7(2.2-10.1)$ & $<0.001$ & 7.69 (3.35-17.6) & $<0.001$ \\
\hline 4 & $10.4(3.4-31)$ & & $24.7(8.1-75.3)$ & \\
\hline Grade 1 & 1 & 0.143 & 1 & 0.048 \\
\hline 2 & $1.89(0.8-4.45)$ & 0.001 & $2.92(1.01-8.46)$ & 0.001 \\
\hline 3 & $4.4(1.78-11)$ & & $6.69(2.25-19.91)$ & \\
\hline Cytology & 1 & 0.022 & 1 & 0.039 \\
\hline Negative & $2.19(1.1-4.3)$ & & $1.98(1.03-3.81)$ & \\
\hline \multicolumn{5}{|l|}{ Positive } \\
\hline LVSI & 1 & 0.032 & 1 & 0.044 \\
\hline Negative & $1.84(1.05-3.2)$ & & $1.81(1.01-3.24)$ & \\
\hline \multicolumn{5}{|l|}{ Positive } \\
\hline Manipulator & 1 & 0.344 & 1 & 0.234 \\
\hline Not used & $1.28(0.76-2.1)$ & & $1.36(0.81-2.29)$ & \\
\hline \multicolumn{5}{|l|}{ Used } \\
\hline Adjuvant treatment & 1 & 0.16 & 1 & 0.018 \\
\hline Not used & $0.59(0.29-1.2)$ & & $0.46(0.24-0.87)$ & \\
\hline \multicolumn{5}{|l|}{ Used } \\
\hline \multicolumn{5}{|l|}{ Subgroup Analysis } \\
\hline Age & $1.06(0.9-1.08)$ & 0.1 & $1.01(0.9-1.05)$ & 0.5 \\
\hline
\end{tabular}

Cl, Confidence interval; LVSI, lymph vascular space invasion. 


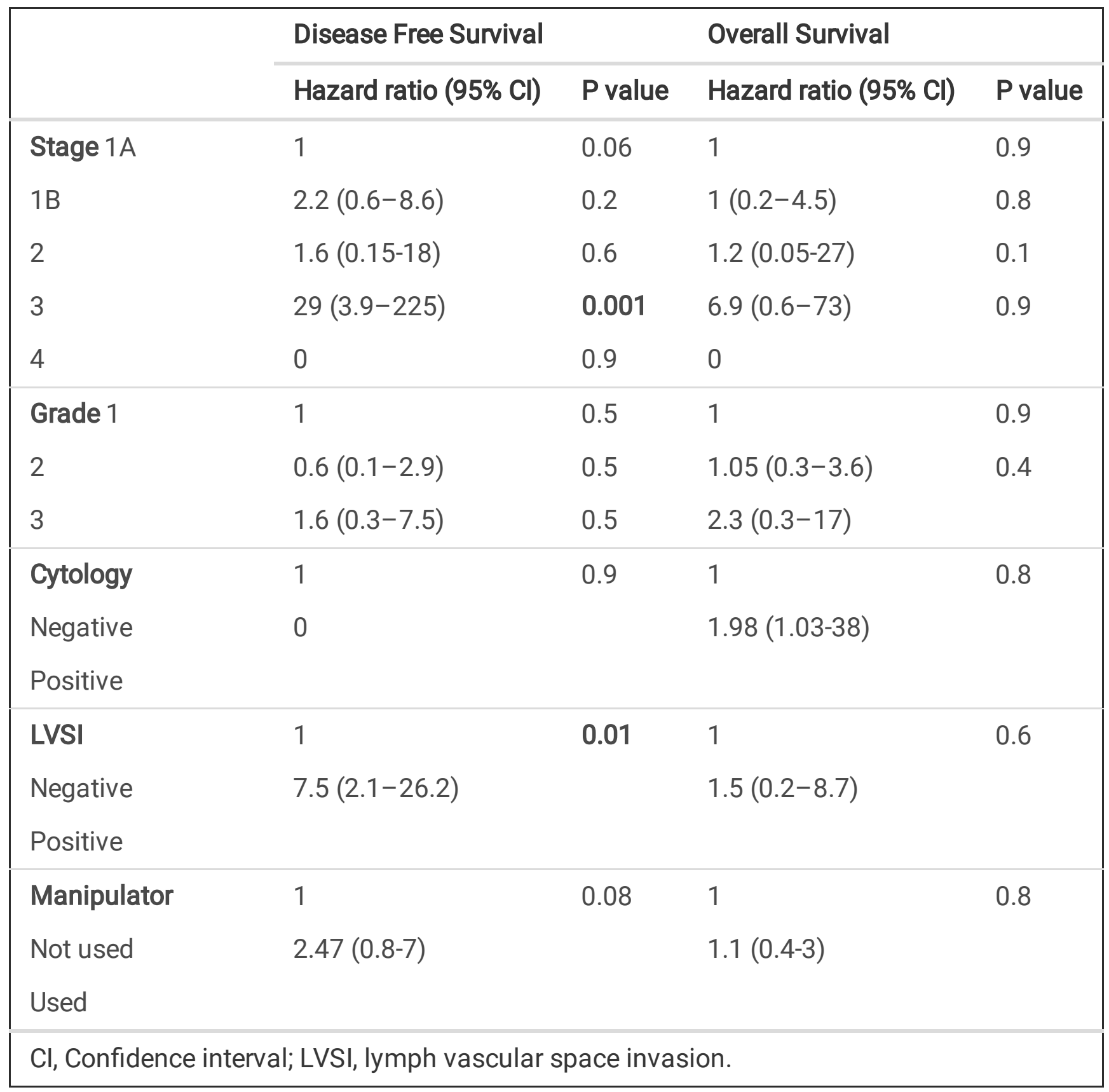

\section{Discussion}

Concerns remain that the introduction of a device into the endometrial cavity may cause disruption of endometrial cancer cells and affect patient's outcome. Available data is limited and indicates that this type of disruption does not seem to have clinically observable adverse effects [12-15]. Data regarding the possible effect of the use of intrauterine manipulators on the long-term outcome of patients with endometrial cancer is scarce. As a result, debates still take place in the gynecologic oncology community raising concern about the use of these devices. Two previous studies have tried to analyse survival after laparoscopic surgery for endometrial cancer using a uterine manipulator. However, those were largely underpowered to investigate long-term outcomes ( $<70$ patients included in each group) $[16,17]$. In a multicenter study by the Italian society of gynecological endoscopy, authors reviewed data from 951 consecutive patients who underwent laparoscopic surgery for endometrial cancer with or without 
intrauterine manipulator. In this study, data regarding the effect of the manipulator on the rate of positive cytology and LVSI was not reported but results showed a non-significant difference in the recurrence rate $(13.5 \%$ vs. $11.6 \%)$ between the manipulator and no manipulator groups [15]. Results of our study raise some safety concerns regarding the use of uterine manipulators during MIS for endometrial cancer. For the entire cohort, total recurrence rate was similar between the two comparison groups but with higher rates of local vaginal recurrence for patients in whom a uterine manipulator was used during surgery. After excluding patients who received adjuvant treatment, the rate of vaginal vault recurrence and total recurrence was significantly higher with a worse DFS in the intra-uterine manipulator group compared to the no manipulator group. However, the use of a uterine manipulator was not found to be a predictor of worse outcome on multivariate analysis that was performed for the entire cohort but was close to statistical significance when performed for the subgroup of patients who did not receive adjuvant treatment.

A recent multicenter study by the Spanish society of gynecology and obstetrics, followed over 2000 women with uterus confined endometrial cancer who underwent minimally invasive surgery and found, in concordance with the results presented here, that patients that were operated using a uterine manipulator had worse oncological outcome [18].

A possible explanation might be that local recurrence may be salvageable by either surgery or radiotherapy, with good long term outcomes [19]. Following recurrence, most patients in our cohort were either operated or received radiotherapy with or without chemotherapy. This is also in accordance to the previously reported LAP2 study that found a potential increased risk of uterine cancer recurrence with manipulator assisted laparoscopic hysterectomies compared to hysterectomies via laparotomy but this risk was quantified and found to be small [7]. We can speculate that the effect of higher recurrence rates on long term outcome of patients in the manipulator group, was eventually diluted by additional treatment modalities and that the presence of a uterine manipulator during surgery did not negatively affect the outcome of endometrial cancer patients on multivariate analysis.

Our results show that the use of an intra-uterine manipulator in MIS for endometrial cancer was not associated with an increase in rates of LVSI, however, we found higher incidence of positive cytology in patients for whom a uterine manipulator was used during surgery. Previously published studies regarding LVSI and positive peritoneal cytology are conflicting, with some suggesting that the use of manipulators does not affect positive peritoneal cytology and/or LVSI [20-24], while other studies show an association between uterine manipulator use and positive peritoneal cytology and/or LVSI [25, 26]. LVSI was previously shown to be an independent adverse prognostic factor for extra-uterine disease, particularly pelvic lymph node metastasis [27, 28], isolated para-aortic lymph node Metastasis [29, 30], and distant hematogenous recurrences [31]. LVSI in the presence of a uterine manipulator may reflect true invasion, pseudo-invasion, or an artifact of manipulator use that resulted in cell displacement into the vessels via a closed pressure system [32]. In the current study, LVSI was found in $27 \%$ of patients, which is in agreement previously published data. According to our results, the use of intrauterine manipulator was not associated with a significant difference in the rate of LVSI. 
With regard to positive cytology, we found that the rate of positive cytology was significantly higher in the group of patients who had an intra-uterine manipulator. Positive cytology was an independent prognostic factor for both DFS and OS on univariate and multivariate analysis. Other reports showed similar results that peritoneal washings were significantly more likely to be positive in women in whom a uterine manipulator had been used $[25,26,33]$. To elucidate whether the use of intra-uterine manipulator increases the risk of positive cytology, its necessary to obtain cytology twice in the same individual, before and after the insertion of the intra-uterine manipulator and compare them.

The current study offers several strengths; data was collected from a large cohort of patients who underwent surgery by a team of surgeons who share a joint surgical protocol and unified approach. This enhances the impact of the use of intrauterine manipulators and allows the current comparison. Moreover, in our study we were also able to perform a subgroup analysis that excludes the effect of adjuvant radiation treatment on the outcome measures. In addition, the follow up time in our study is relatively long and was previously shown to include over $90 \%$ of recurrences from initial treatment [34]. However, this study is not without limitations including the inherent limitations that results from its retrospective nature. In addition, we did not perform coagulation of the tubes at the beginning of the procedure and this might have an effect with regard to positive cytology.

Finally, in the current study we deal with the long-term outcome of uterine manipulators in endometrial cancer, but can we extrapolate these results to cervical cancer? Could the data presented here provide some hints to explain the poorer results for cervical cancer patients undergoing minimal invasive surgery with a uterine manipulator. Several theories have been proposed to explain the unexpected results of the LACC trial that compared minimally invasive radical hysterectomy to open abdominal radical hysterectomy among women with early-stage cervical cancer. One theory suggests that the use of a uterine manipulator is a possible cause of the poorer oncological outcome after minimal invasive radical hysterectomy for cervical cancer [35]. The results presented here show higher recurrence rates, mainly at the vaginal vault and decreased disease-free survival in a subgroup of patients who were operated using an intra-uterine manipulator and did not receive adjuvant treatment. In contrast to cervical cancer, the rise in disease recurrence did not significantly affect OS. We can speculate that this could either be related to differences in the biology of squamous cell carcinomas of the cervix and endometrial cancers, and/or be due to the salvage potential of the treatment modalities for recurrent localized endometrial cancer. $A$ recent international European observational study comparing minimally invasive surgery versus open abdominal radical hysterectomy in patients with stage IB1 cervical cancer found that patients that underwent minimally invasive surgery using a uterine manipulator had a 2.76-times higher hazard of relapse and those without the use of a uterine manipulator had similar disease-free-survival to the open surgery group [36].

In conclusion, results of the current study show that the use of an intra uterine manipulator is associated with a minimal impact on the oncological outcomes of patient who undergo MIS and adjuvant treatment for endometrial cancer, but may have implications on outcome for low risk patients who do not receive adjuvant treatment.

Page $16 / 21$ 


\section{Declarations}

Consent to participate not applicable.

Funding: not applicable.

Conflicts of interest/Competing interests: The authors report no conflict of interest.

Ethics approval: Ethical approval was waived by the local Ethics Committee of University A in view of the retrospective nature of the study and all the procedures being performed were part of the routine care.

Acknowledgement: this work was supported by grants from the Israel Cancer Research Fund and the Gloria's Girls.

\section{CRediT authorship contribution statement:}

Ido Laskov: Conceptualization, Methodology, Software, Validation, Formal analysis, Investigation, Resources, Data curation, Writing - original draft, Writing - review \& editing, Visualization, Supervision, Project administration. Nadav Michaan: Methodology, Software, Validation, Formal analysis, Investigation, Data curation, Writing - original draft, Visualization. Liron Kogan: Conceptualization, Investigation, Data curation, Writing - review \& editing. Xing Zeng: Resources, Investigation, Data curation, Writing - review \& editing. Shannon Salvador: Resources, Investigation, Data curation, Writing - review \& editing. Kris Jardon: Resources, Investigation, Data curation, Writing - review \& editing. Susie Lau: Resources, Investigation, Data curation, Writing - review \& editing. Lucy Gilbert: Resources, Investigation, Data curation, Writing - review \& editing, Supervision. Walter H. Gotlieb: Conceptualization, Methodology, Investigation, Resources, Data curation, Writing - original draft, Writing - review \& editing, Supervision. Roy Kessous: Conceptualization, Methodology, Validation, Formal analysis, Investigation, Data curation, Writing - original draft, Writing - review \& editing, Visualization.

\section{References}

1. Creutzberg CL et al (2000) Surgery and postoperative radiotherapy versus surgery alone for patients with stage-1 endometrial carcinoma: multicentre randomised trial. PORTEC Study Group. Post Operative Radiation Therapy in Endometrial Carcinoma. Lancet 355(9213):1404-1411

2. Keys HM et al (2004) A phase III trial of surgery with or without adjunctive external pelvic radiation therapy in intermediate risk endometrial adenocarcinoma: a Gynecologic Oncology Group study. Gynecol Oncol 92(3):744-751

3. Group AES et al (2009) Adjuvant external beam radiotherapy in the treatment of endometrial cancer (MRC ASTEC and NCIC CTG EN.5 randomised trials): pooled trial results, systematic review, and meta-analysis. Lancet 373(9658):137-146

4. Creutzberg CL et al (2011) Fifteen-year radiotherapy outcomes of the randomized PORTEC-1 trial for endometrial carcinoma. Int J Radiat Oncol Biol Phys 81(4):e631-e638 
5. Patel PR et al (2014) Disparities in use of laparoscopic hysterectomies: a nationwide analysis. J Minim Invasive Gynecol 21(2):223-227

6. Walker JL et al (2009) Laparoscopy compared with laparotomy for comprehensive surgical staging of uterine cancer: Gynecologic Oncology Group Study LAP2. J Clin Oncol 27(32):5331-5336

7. Walker JL et al (2012) Recurrence and survival after random assignment to laparoscopy versus laparotomy for comprehensive surgical staging of uterine cancer: Gynecologic Oncology Group LAP2 Study. J Clin Oncol 30(7):695-700

8. van den Haak L et al (2015) Efficacy and safety of uterine manipulators in laparoscopic surgery: a review. Arch Gynecol Obstet 292(5):1003-1011

9. Nassif J, Wattiez A (2010) Clermont Ferrand uterine manipulator. Surg Technol Int 20:225-231

10. Peeters $F$ et al (2010) Technical modifications in the robotic-assisted surgical approach for gynaecologic operations. Journal of Robotic Surgery 4(4):253-257

11. Benedet JL et al (2000) FIGO staging classifications and clinical practice guidelines in the management of gynecologic cancers. FIGO Committee on Gynecologic Oncology. Int J Gynaecol Obstet 70(2):209-262

12. Eltabbakh GH, Mount SL (2006) Laparoscopic surgery does not increase the positive peritoneal cytology among women with endometrial carcinoma. Gynecol Oncol 100(2):361-364

13. Polyzos NP et al (2010) Intraperitoneal dissemination of endometrial cancer cells after hysteroscopy: a systematic review and meta-analysis. Int J Gynecol Cancer 20(2):261-267

14. Shinohara S et al (2017) Risk of spilling cancer cells during total laparoscopic hysterectomy in lowrisk endometrial cancer. Gynecol Minim Invasive Ther 6(3):113-115

15. Uccella $S$ et al., The effect of a uterine manipulator on the recurrence and mortality of endometrial cancer: a multi-centric study by the Italian Society of Gynecological Endoscopy. Am J Obstet Gynecol, 2017. 216(6): p. 592 e1-592 e11

16. Marcos-Sanmartin J et al (2016) Does the Type of Surgical Approach and the Use of Uterine Manipulators Influence the Disease-Free Survival and Recurrence Rates in Early-Stage Endometrial Cancer? Int J Gynecol Cancer 26(9):1722-1726

17. Tinelli $R$ et al (2016) Laparoscopic treatment of early-stage endometrial cancer with and without uterine manipulator: Our experience and review of literature. Surg Oncol 25(2):98-103

18. Padilla-Iserte $\mathrm{P}$ et al., Impact of uterine manipulator on oncological outcome in endometrial cancer surgery. Am J Obstet Gynecol, 2020

19. Hardarson HA et al (2015) Vaginal vault recurrences of endometrial cancer in non-irradiated patients - Radiotherapy or surgery. Gynecol Oncol Rep 11:26-30

20. Frimer $\mathrm{M}$ et al (2010) Micrometastasis of endometrial cancer to sentinel lymph nodes: is it an artifact of uterine manipulation? Gynecol Oncol 119(3):496-499

21. Lee M et al (2013) Effects of uterine manipulation on surgical outcomes in laparoscopic management of endometrial cancer: a prospective randomized clinical trial. Int J Gynecol Cancer 
23(2):372-379

22. Momeni $\mathrm{M}$ et al (2013) Does the type of surgery for early-stage endometrial cancer affect the rate of reported lymphovascular space invasion in final pathology specimens? Am J Obstet Gynecol 208(1):71 e1-e6

23. Machida $\mathrm{H}$ et al (2018) Intrauterine Manipulator Use During Minimally Invasive Hysterectomy and Risk of Lymphovascular Space Invasion in Endometrial Cancer. Int J Gynecol Cancer 28(2):208-219

24. Machida $\mathrm{H}$ et al (2016) Timing of Intrauterine Manipulator Insertion During Minimally Invasive Surgical Staging and Results of Pelvic Cytology in Endometrial Cancer. J Minim Invasive Gynecol 23(2):234-241

25. Delair $D$ et al (2013) Tumoral displacement into fallopian tubes in patients undergoing robotically assisted hysterectomy for newly diagnosed endometrial cancer. Int J Gynecol Pathol 32(2):188-192

26. Krizova A et al (2011) Histologic artifacts in abdominal, vaginal, laparoscopic, and robotic hysterectomy specimens: a blinded, retrospective review. Am J Surg Pathol 35(1):115-126

27. Akbayir $O$ et al (2012) The novel criteria for predicting pelvic lymph node metastasis in endometrioid adenocarcinoma of endometrium. Gynecol Oncol 125(2):400-403

28. Zhang C, Wang C, Feng W (2012) Clinicopathological risk factors for pelvic lymph node metastasis in clinical early-stage endometrioid endometrial adenocarcinoma. Int J Gynecol Cancer 22(8):13731377

29. Chang SJ et al (2011) Lymph-vascular space invasion as a significant risk factor for isolated paraaortic lymph node metastasis in endometrial cancer: a study of 203 consecutive patients. Ann Surg Oncol 18(1):58-64

30. Park JY et al (2010) The role of pelvic and/or para-aortic lymphadenectomy in surgical management of apparently early carcinosarcoma of uterus. Ann Surg Oncol 17(3):861-868

31. Gadducci A et al (2009) Lymph-vascular space involvement and outer one-third myometrial invasion are strong predictors of distant haematogeneous failures in patients with stage I-II endometrioid-type endometrial cancer. Anticancer Res 29(5):1715-1720

32. Logani S et al (2008) Vascular "pseudo invasion" in laparoscopic hysterectomy specimens: a diagnostic pitfall. Am J Surg Pathol 32(4):560-565

33. Zhang $C$ et al (2014) Relationship between minimally invasive hysterectomy, pelvic cytology, and lymph vascular space invasion: a single institution study of 458 patients. Gynecol Oncol 133(2):211-215

34. Sohaib SA et al (2007) Recurrent endometrial cancer: patterns of recurrent disease and assessment of prognosis. Clin Radiol 62(1):28-34; discussion 35 - 6

35. Ramirez PT et al (2018) Minimally Invasive versus Abdominal Radical Hysterectomy for Cervical Cancer. N Engl J Med 379(20):1895-1904

36. Chiva L et al (2020) SUCCOR study: an international European cohort observational study comparing minimally invasive surgery versus open abdominal radical hysterectomy in patients with stage IB1 
Figures

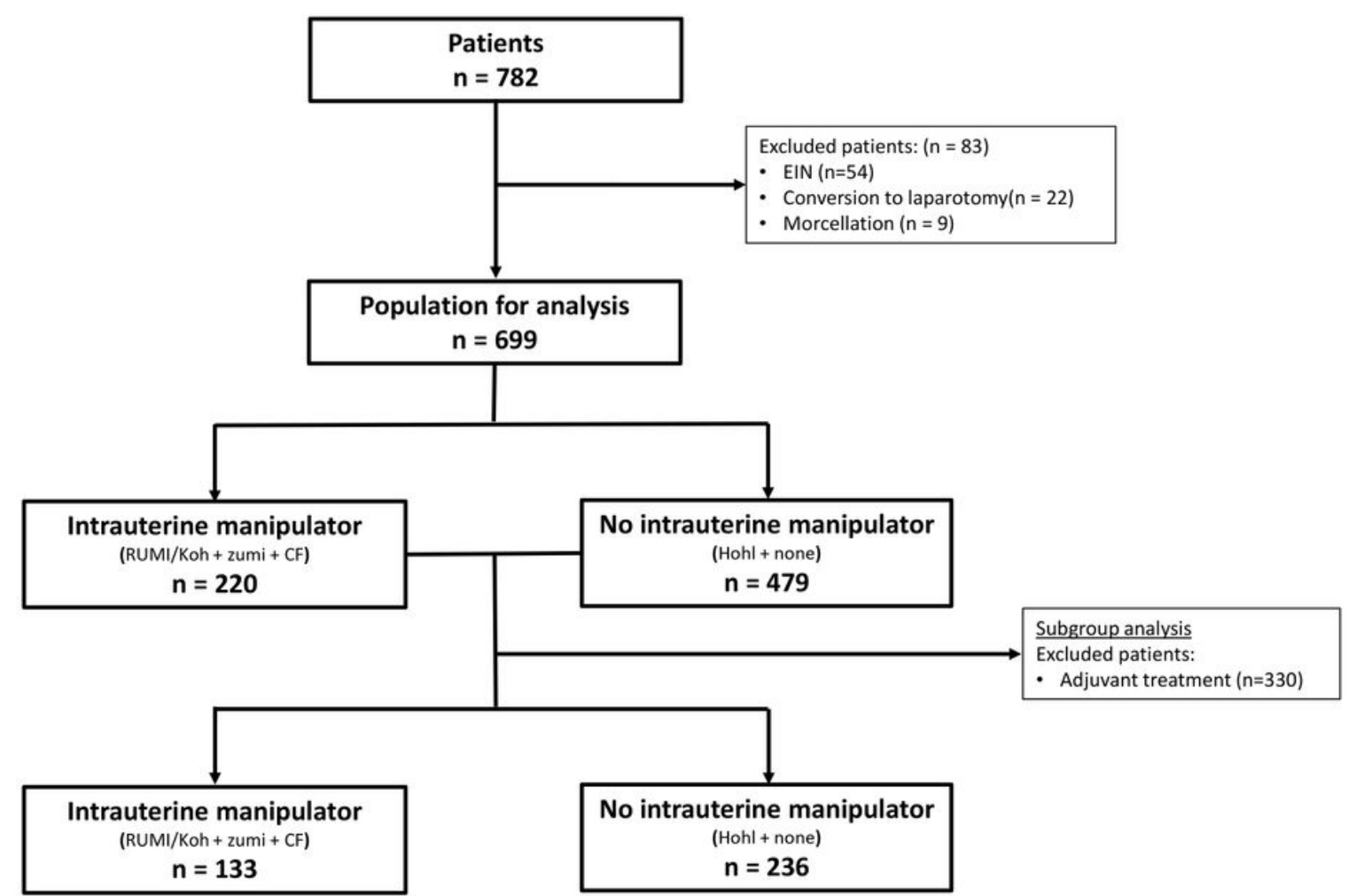

Figure 1

Distribution of patients eligible for analysis. 

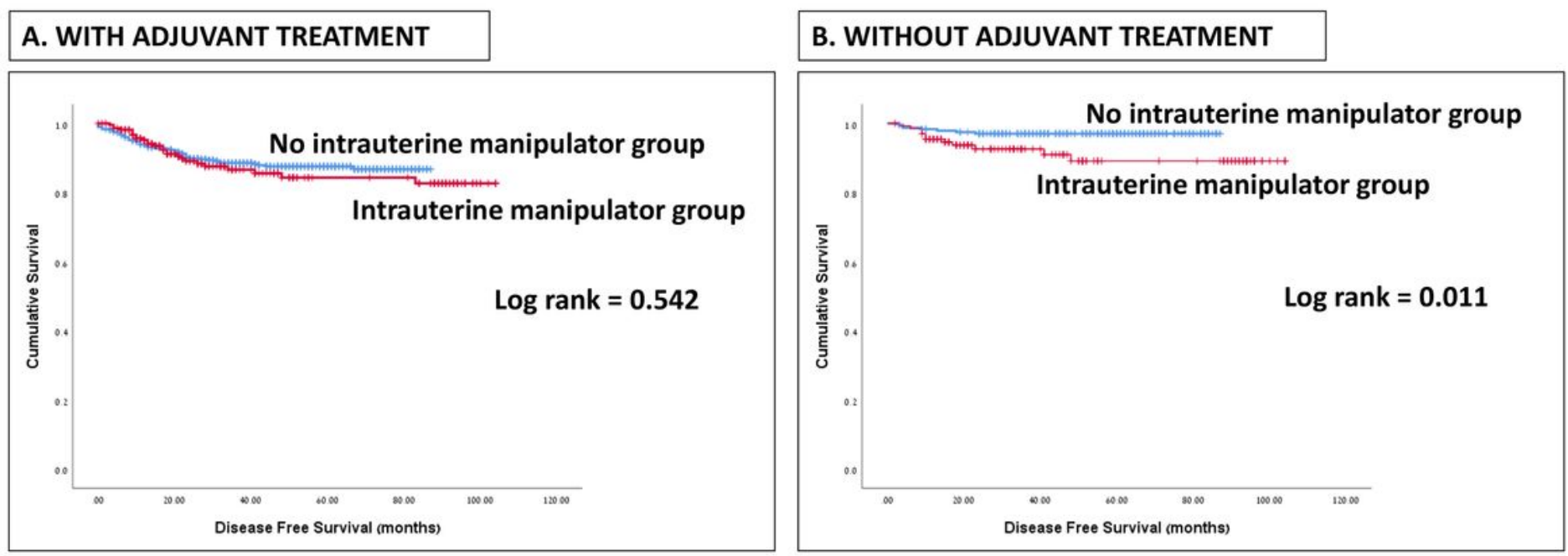

\section{Figure 2}

Disease-free survival, Kaplan-Meyer curves compare disease-free survival in the manipulator vs no manipulator groups, for patients who received adjuvant treatment $(A)$ and patients that did not receive adjuvant treatment (B). 